\title{
Implementing Telemedicine in Medical Emergency Response: Concept of Operation for a Regional Telemedicine Hub
}

\author{
Wei Xiong • Aaron Bair • Christian Sandrock • \\ Sophia Wang • Javeed Siddiqui • Nathaniel Hupert
}

Received: 24 August 2010 /Accepted: 5 November 2010 /Published online: 14 December 2010

(C) The Author(s) 2010. This article is published with open access at Springerlink.com

\begin{abstract}
A regional telemedicine hub, providing linkage of a telemedicine command center with an extended network of clinical experts in the setting of a natural or intentional disaster, may facilitate future disaster response and improve patient outcomes. However, the health benefits derived from the use of telemedicine in disaster response have not been quantitatively analyzed. In this paper, we present a general model of the application of telemedicine to disaster response and evaluate a concept of operations for a regional telemedicine hub, which would create distributed surge capacity using regional telemedicine networks connecting available healthcare and telemedicine infrastructures to external expertise. Specifically, we investigate (1) the scope of potential use of telemedicine in disaster response; (2) the operational characteristics of a regional telemedicine hub using a new discrete-event simulation model of an earthquake scenario; and (3) the benefit that the affected population may gain from a coordinated regional telemedicine network.
\end{abstract}

W. Xiong $\cdot$ N. Hupert $(\bowtie)$

Departments of Public Health and Medicine,

Weill Medical College, Cornell University,

New York, NY, USA

e-mail: nah2005@med.cornell.edu

A. Bair $\cdot$ C. Sandrock $\cdot$ J. Siddiqui

University of California-Davis,

Sacramento, CA, USA

S. Wang

Cayuga Partners,

Ithaca, NY, USA

N. Hupert

NewYork-Presbyterian Hospital,

New York, NY, USA
Keywords Telemedicine $\cdot$ Disaster response $\cdot$ Discrete-event simulation · Emergency medicine $\cdot$ Earthquake

\section{Introduction}

Natural and intentional disasters can unfold quickly and cause a variety of injuries to a large number of affected individuals, necessitating both immediate and sustained medical care. While the timely extrication, stabilization, and transport of injured victims of mass casualty incidents is a cornerstone of emergency medical and trauma care, medical responses are often impeded by overwhelming numbers of patients and the limited number of available medical personnel and resources, resulting in delayed treatment [25]. Major disasters such as earthquakes or hurricanes may also damage infrastructure within the affected area, such as communications facilities and roads, further impeding the delivery of medical personnel and material resources from external sources, including neighboring communities, humanitarian organizations, and State and federal sources.

The fundamental premise of telemedicine is that voice and data linkages permit medical services to be provided remotely. When equipped with basic telecommunication devices that can be deployed by mobile units, responders at the scene of a disaster can quickly establish telemedicine linkages. This would potentially increase both the speed and the capacity of medical response and make it available when and where it is needed. Applications of telemedicine to disaster response began in the mid-1980s. Following the devastating 1985 Mexico City earthquake, NASA provided advanced satellite communication technology to support the international relief and rescue operations [27]. After the 1988 Armenian earthquake, telemedicine was employed to 
provide clinical consultation to several regional hospitals via the U.S.-U.S.S.R. Space Bridge project $[2,27]$. In this decade, telemedicine has been more widely used in various ways in response to disasters including earthquakes, tsunami and hurricanes $[1,10,13,14,24]$. In addition to response to real disasters, numerous telemedicine experiments, exercises and simulations of "staged" disasters have been carried out worldwide to evaluate the usefulness and performance of telemedicine systems $[12,20]$.

Many in the emergency medicine and preparedness community believe that, based on the observed value of existing telemedicine capabilities for disaster management, more advanced telemedicine systems will greatly facilitate disaster response. However, support for this belief is mainly based on expert opinions, case studies, or anecdotal examples. Many questions arise as to how to most effectively apply and integrate telemedicine into a regional response framework. For example, what is the role of telemedicine in existing protocols and guidelines for disaster response? How can external physicians and other resources be mobilized in such an incident through the use of telemedicine? What are the appropriate infrastructure and information systems to support telemedicine interventions in the event of a major disaster?

To address these issues in a quantitative fashion, we examined whether the concept of a regional telemedicine hub (TMH) is the best organizational model to enable efficient, effective, and equitable delivery of medical services to a target population in the aftermath of a major medical disaster. The establishment of a telemedicine network with a regional hub has significant policy implications, such as the coordinated selection of communication platforms and information systems, the consolidated management of resources in a target area, and the facilitation of NIMS (National Incident Management System)-compliant centralized command and control centers to direct the healthcare response for disasters. However, while a regional telemedicine hub with an extended network has the potential to alleviate multiple problems during disaster response, there is no consensus about how to quantify the health-related benefit associated with the proposed organization model [8]. In this paper, we describe a comprehensive quantitative analysis that assesses the benefits of the telemedicine hub concept in emergency response for a hypothetical earthquake scenario.

\section{Methods}

We designed a comparative simulation study to examine one of the potential applications of regional telemedicine network to disaster response, the augmentation of local medical surge capacity. The scenarios concentrate on the early phases of hospital patient management. We explore the impact of using telemedicine to provide emergency specialty care to patients at local hospitals on medical surge capacities at both local and regional levels. We aim to (1) provide a conceptual framework to incorporate telemedicine into emergency response; and (2) determine where it is appropriate to apply quantitative analysis to improve the effectiveness of disaster response activities, potentially measured through such factors as treatment capability and time to definitive treatment.

\section{Modeling approach}

We examine and compare two cases: a base case representing the current process of the acute phase of hospital-based medical response to mass casualties caused by an earthquake; and a second, alternative telemedicine case for which the response process allows and supports telemedicine interventions at the local level. Simulation environments were developed for both cases, which consist of (1) probabilistic models to generate the earthquake injury profile; (2) queuing models to represent the delay of treatment when injured patients seek care at local Emergency Departments (ED), local specialty care, transportation from local hospitals to the Designated Receiving Center (DRC) and specialty care at the center; and (3) probabilistic mortality models to simulate potential patient deaths that are related to delay of treatment. These models were created using the ARENAC simulation package (Version 12.0).

The response processes for the two cases are illustrated in Figs. 1 and 2. Each simulation creates patient flow through the telemedicine system and calculates potential mortality associated with treatment delay along the resulting patient management paths. In both cases, patients with multiple types of injuries are generated via a probabilistic casualty generation model to induce the distributions of patient arrival, injury type, injury severity, etc. based on factors such as the scale of the disaster and local population distribution. Patients are sent to local hospitals for treatment, where emergency physicians examine the patients to identify their injury type and condition and perform routine or resuscitative procedures when necessary. Most patients are discharged (released or hospitalized for further treatment) after local ED care and would not require a specialist care during the simulation time horizon. However, some patients with a critical condition will require consultation or specialty care that may not be accessible at local treatment facilities. For this group of patients there are two possible pathways: if a specialist in the required area is present at the local hospital they will be called to assist with care; otherwise if a specialist is 


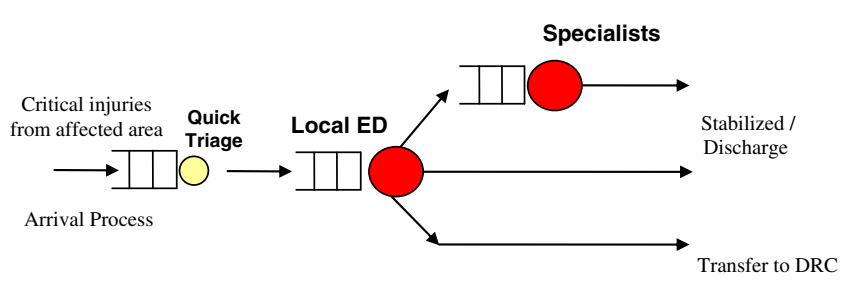

Death may happen at any point in time

Fig. 1 Current hospital-based medical response process (Base Case)

unavailable (or too busy) at the local site, the emergency physician will have to transfer the patient to a regional DRC that provides specialty care.

Queuing models are used to represent both patient waiting and treatment processes at local EDs, local specialty care if available, transportation to the DRC, and specialty care at the DRC. Due to surging demand and limited resources at both local hospitals and at the center, patients will wait for ED and specialty care as well as transportation at local sites, and for specialty care at the DRC. Priority is given to patients in critical condition at local and DRC ED queues. "Balking" (i.e. leaving prior to treatment) is possible at local ED queues. For example, if an incoming patient requires specialist consultation but finds the current queue length for ED beds exceeded a threshold value, a transfer decision may be made to send the patient to the receiving center even when a specialist is available at the local facility. Based on our review of relevant studies, we use three time dependent patient survival curves throughout the process to determine the risk of death due to prolonged waiting $[6,9,22,23]$.

Under all three mortality assumptions increasing local treatment capacity and reducing delay to treatment would decrease mortality. We explored how such changes can be rapidly achieved through introducing telemedicine interventions to local care providers during these decisive moments. We assumed that remote generalist emergency medical providers could be connected to local care centers through the regional DRC. In addition, patients at the local care site who require more advanced care could access specialists via the telemedicine link, thus avoiding the need for immediate transportation to the DRC before the patient is properly stabilized. In this case the receiving center also serves as a control hub that facilitates and supports telemedicine calls between local healthcare facilities and remote specialists. However, since the number of simultaneous telemedicine sessions is constrained by available remote specialists as well as the center's technical capacity, it is possible for patients to have to wait for a telemedicine specialist or a telelink at local hospitals during times of peak demand.
Data and assumptions

The simulated disaster scenario is an earthquake that results in large numbers of casualties. The simulation focuses on the early phase of the disaster, specifically, the hospitalbased medical response dealing with injuries that arrive within the first $48 \mathrm{~h}$ following the disaster. Other disaster response efforts, such as search and rescue, and the recovery of infrastructure, are not explicitly represented in the simulation. Later phases of on-going medical support, such as follow-up care and rehabilitation, are also outside the immediate scope of this study. Data required for the comparative simulation study are collected and generated as follows:

(1) Earthquake scale and patient volume: Historical data suggests that the volume of casualties resulting from an earthquake may vary significantly depending upon the scale, time and location of onset of the disaster [15]. For simplicity, we adopt a ratio of affected population to generate the patient volume. We consider earthquakes ranging from minor (with several hundred injuries) to medium and major scale earthquakes (with thousands of injured patients). We assume casualty ratios of $0.1 \%$ for minor, $0.2 \%$ for medium, and $0.5 \%$ for the major scenario.

(2) Simulation time horizon: The time horizon specified for the simulation study is selected to deal with injuries that arrive within the first $48 \mathrm{~h}$ following the onset of the disaster, as suggested by past experience and literature. In the 1994 Northridge earthquake, most injuries were hospitalized during the first 2 days after it occurred $[5,18]$. We also assume that patient arrival process in the simulation time interval is a Poisson process.

(3) Impacted regions: For purposes of population impact, we centered the simulated earthquake on the San

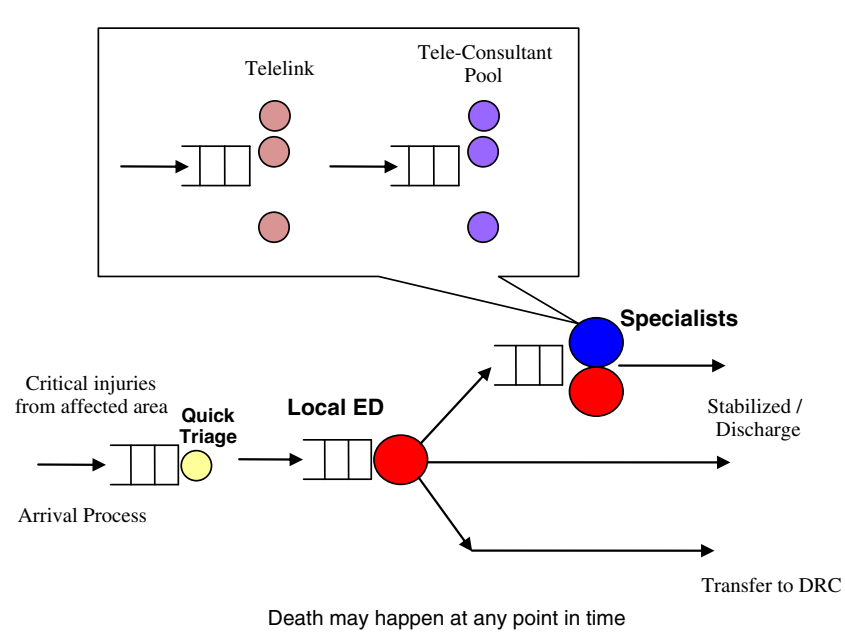

Fig. 2 Telemedicine-enhanced hospital-based medical response 
Francisco Bay/Oakland Area. Some of the casualties from this region may be assigned and dispatched to a DRC which lies outside the multiple faults around the San Francisco/Oakland area and would be more likely to be structurally intact. The simulation considers a portion of the potentially impacted region, with a total population of $\sim 540,000$ over four local areas, with an average transportation time to the DRC of approximately $30 \mathrm{~min}$. We also assume there are 4 local treatment facilities available for emergency medical services in the affected area.

(4) Injury types: Three injury types are explicitly considered in the simulation, i.e. trauma, burn, and "other", to represent the most commonly observed earthquakerelated injuries that often require specialty care.

(5) Injury condition: Based on observations obtained in past mass casualty events $[5,16,18]$, we estimate the percentage of severely injured patients to be $20 \sim 30 \%$. In this study we occasionally use "critical condition" to indicate this group of patients, which is not to be confused with the medical term used in the field referring to patients with unstable vital signs. These patients plus selected "non-critical" patients are considered to require emergency specialist care for the purpose of the simulation.

(6) Hospital capacity: The local hospitals' capacities (as represented by the number of local ED beds in the simulation), as well as capacity for the DRC, are obtained based on state average levels [15]. The numbers of specialists available at both local and regional levels are hypothetical and are estimated based on surveys obtained from the region in this reference [15]. Table 1 displays information on local demographics and local hospitals' capacity that is used in the simulation:

(7) Specialty treatment: We estimate the treatment time of initial specialty care to be 40-60 min, which may implicitly include resuscitation, stabilization, triage, procedural supervision, and excludes continuing care (i.e., routine intensive care unit care) due to the scope of the simulation.

(8) Patient mortality: We modeled patient mortality as a function of time left waiting for definitive stabilization and medical care (Fig. 3). [9]. Patient mortality is possible at multiple points in the simulation, including: before ED care, after ED and before specialty care, and after specialty care.

(9) Telemedicine capacity: For the telemedicine case, the DRC's technical capacity in terms of supporting telemedicine calls is estimated to have five telelinks working simultaneously. Communication and telemedicine abilities are assumed to be available at local "spoke" sites.

Simulation parameters and outputs

One-hundred simulation replications were conducted for each of the three disaster scenarios in both cases. We collected the following data and performance metrics from the simulation:

(1) Average number of injuries generated (by type and condition).

(2) Average number of patients (by type and condition) that receive local ED care, receive local specialty care, are transferred to DRC, and receive DRC specialty care.

(3) Average patient mortality (by type and condition) before local ED (waiting for ED bed and treatment), related to transportation (waiting for ambulance and en-route to DRC), and before DRC ED (waiting for ED bed for specialty care).

(4) Average patient waiting time (by type and condition) for bed at local ED, for local specialty care, for transportation to DRC, for DRC ED bed, DRC specialty care, etc.

(5) Resource utilization, including specialist, bed, and telelink.

\section{Results}

Patient arrivals

Patient arrival numbers for the three disaster scenarios (major, medium and minor scale) are summarized in Fig. 4.

Table 1 Basic input data to simulation

\begin{tabular}{|c|c|c|c|c|c|c|c|}
\hline & Population & $\begin{array}{l}\text { Transport time } \\
\text { (min) }\end{array}$ & $\begin{array}{l}\text { Available } \\
\text { ED bed capacity }\end{array}$ & $\begin{array}{l}\text { Available } \\
\text { ambulance }\end{array}$ & $\begin{array}{l}\text { Specialists } \\
\text { (Trauma) }\end{array}$ & $\begin{array}{l}\text { Specialists } \\
\text { (Burn) }\end{array}$ & $\begin{array}{l}\text { Specialists } \\
\text { (Other) }\end{array}$ \\
\hline Local ED 1 & 145,000 & 25 & 6 & 12 & 1 & 0 & 0 \\
\hline Local ED 2 & 75,000 & 40 & 3 & 6 & 0 & 0 & 0 \\
\hline Local ED 3 & 220,000 & 25 & 8 & 16 & 2 & 0 & 1 \\
\hline Local ED 4 & 100,000 & 30 & 4 & 8 & 1 & 0 & 0 \\
\hline DRC & - & - & 20 & - & 5 & 2 & 2 \\
\hline Remote & - & - & - & - & 6 & 2 & 2 \\
\hline
\end{tabular}


Time-Dependent Mortality/Survival for Critically Injured

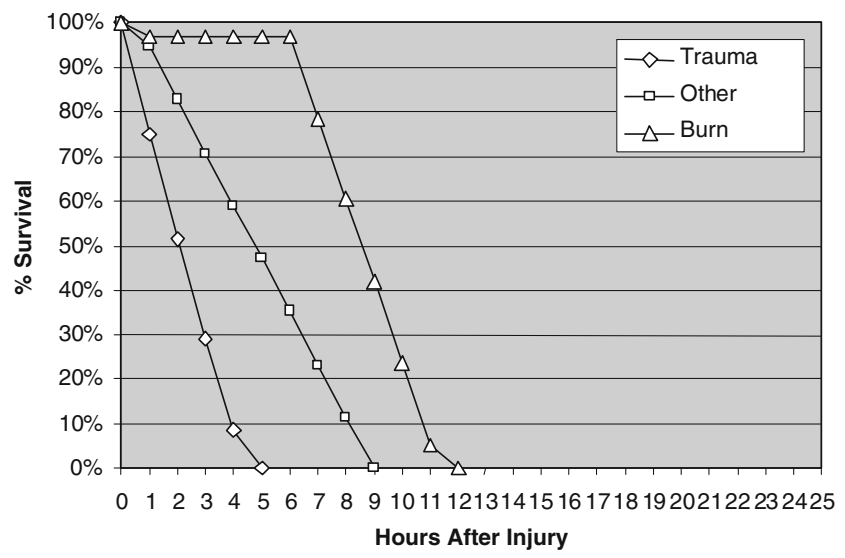

Fig. 3 Time-dependent mortality curves for critically injured

Our simulation of a major earthquake produced on average 2,700 injuries with 1,970 non-critical and 730 critically injured patients. Similarly, there are 1,080 casualties (774 non-critical and 306 critical) in the medium case and 540 casualties (381 non-critical and 159 critical).

\section{Mortality}

Patient mortality outcomes from the three earthquake scenarios are illustrated in Figs. 5 and 6. Compared to the base case representing current practice, the total number of deaths is reduced in the telemedicine case by $5.4 \%, 36.5 \%$ and $27.3 \%$ for the major, medium and minor scale earthquake scenarios respectively. The results suggest that the performance of the telemedicineenhanced medical response process is superior to that of the base process in all three disaster scenarios in terms of patient mortality.

Across the three disaster scenarios, the biggest single location for mortality reduction from using telemedicine occurs at the DRC, by $15.37 \%, 91.69 \%$ and $99.93 \%$ respectively for major, medium, and minor scenarios. This reduction in deaths in the telemedicine case can be explained by the augmented triage that allows more patients to stay local and thus avoid delay of treatment while being transported to the center (where congestion may happen). More specifically, even though a higher percentage of (and in absolute numbers, more) patient deaths happen at the local facilities in the telemedicine cases, significantly fewer occur while waiting for transportation/en-route to the DRC or at the DRC. This indicates that the primary reasons for reduction in total deaths in the telemedicine cases include:

(1) Reduction in the number of patients transferred to avoid extra transportation time;
Fewer patients are transferred in the telemedicine case, especially in the medium and minor scale earthquake scenarios. The reduction in transferred patients is smaller the major earthquake scenario because the transfer decision is made under one of the following two conditions: if a specialist is not available locally, or when there are currently too many people waiting for local ED beds when the patient enters the queue. In the major scale earthquake scenario, the overwhelming number of arriving patients results in long queues at local EDs, forcing some patients to be transferred even when specialists are reachable at the local facility (via telemedicine). This also indicates that local ED beds may be a bottleneck resource that limits the system's overall performance. More details on resource usage and bottleneck analysis, as well as local transfer decisions and rules, is discussed below.

(2) Reduction in transportation-related waiting times;

Most patients that need specialty care are critically injured, and their survival times is modeled to be be significantly shorter than those who are non-critical. When more patients are transferred to the center, as in the current process case, unavoidable transportation time (which takes about 25-40 $\mathrm{min}$ ) and extra waiting time at the receiving center prolongs the patients' total delay before they are able to receive specialty care, resulting in more deaths. In

(3) Reduction in overall congestion at the DRC which leads to reduced patient waiting time.

We observe that in all three disaster scenarios, almost all three types of patients' waiting times for ED bed as well as for specialists at the DRC are reduced in the telemedicine case. The only exception is for Trauma (Type 1) patients in the major scale earthquake scenario. A closer look at the numbers reveals that for the major scale disaster scenario, even though fewer trauma patients are transferred, and fewer arrive dead at the center in the telemedicine case than

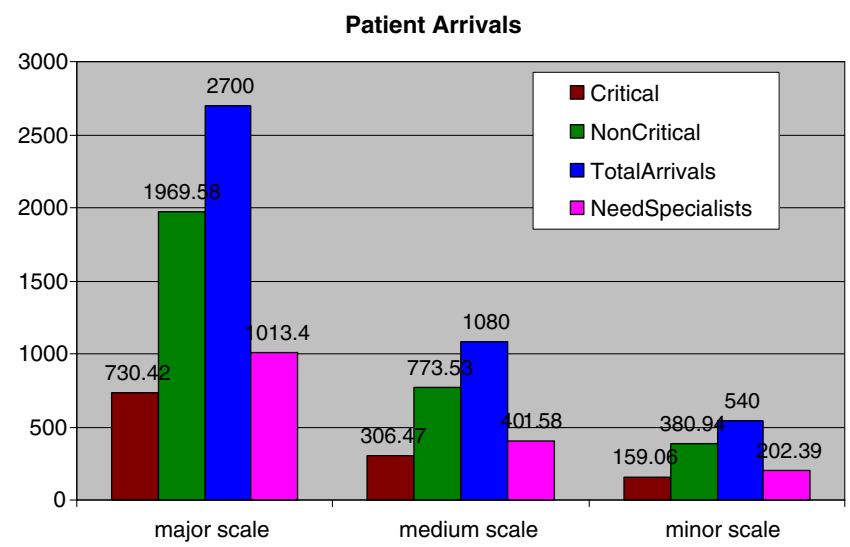

Fig. 4 Average arrivals by patient type for major through minor earthquake scenarios 
Patient Outcome: BaseCase, major scale

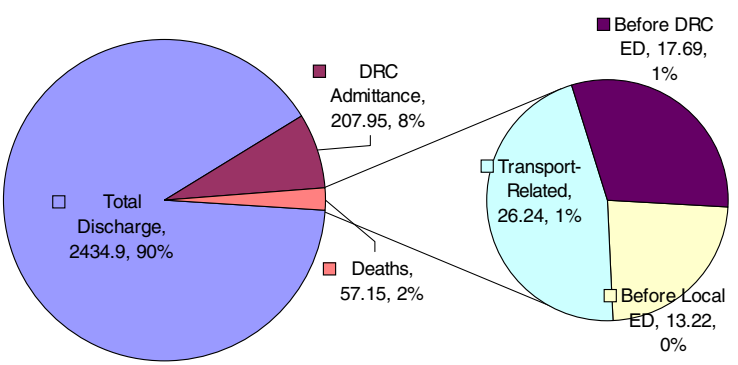

Patient Outcome: BaseCase, medium scale

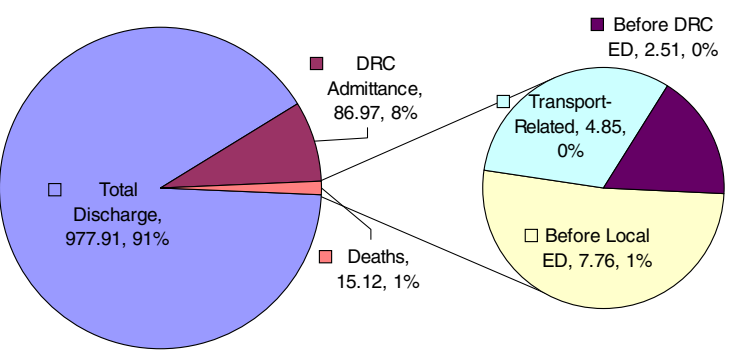

Patient Outcome: BaseCase, minor scale

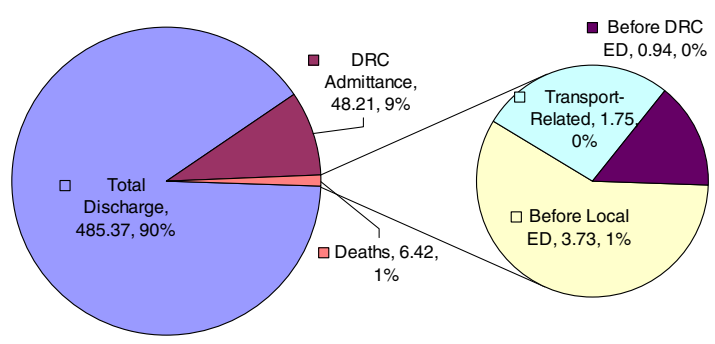

Patient Outcome: Telemedicine, major scale

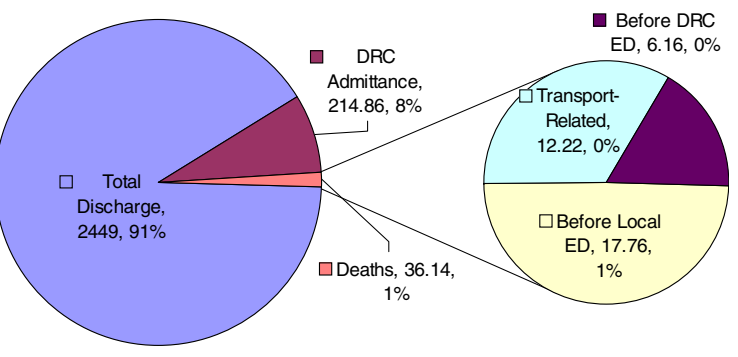

Patient Outcome: Telemedicine, medium scale

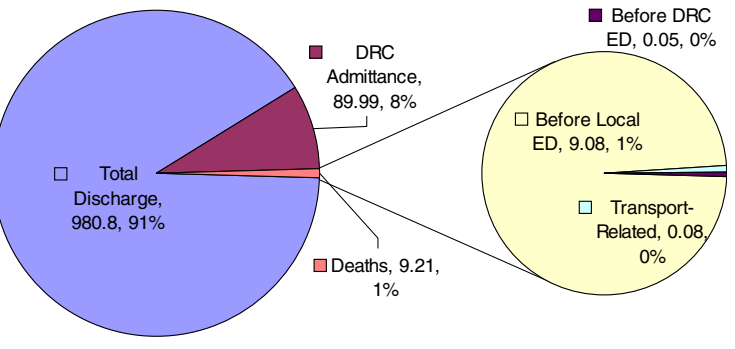

Patient Outcome: Telemedicine, minor scale

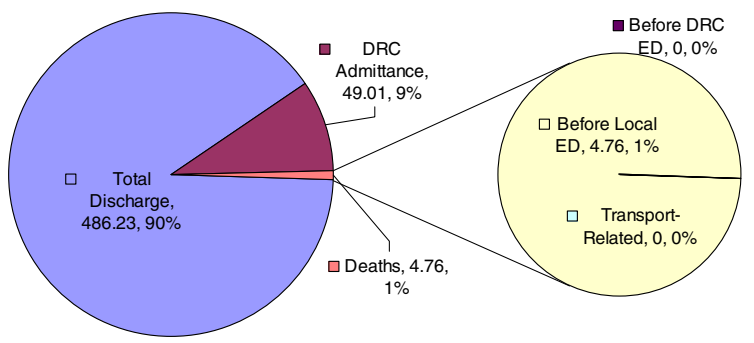

Fig. 5 Average patient mortality outcomes for base and telemedicine scenarios, with major, medium, and minor-scale earthquakes

in the current case, in the end more trauma patients manage to survive until they receive specialty care. The numbers are shown in the Table 2. In other words, in the telemedicine case trauma specialists at the DRC actually spend more time caring for patients that are still alive, resulting in slightly longer average waiting time for subsequent arrivals.. Still, a higher percentage $(60.72 \%$ vs. $57.34 \%)$ of trauma patients are saved in the telemedicine case at the DRC.

\section{Resource utilization}

Resource use estimates from the simulation are summarized in Tables 3 and 4. For the medium and minor scale scenarios, the local EDs' bed usage rates are typically lower in the telemedicine case than in the base case (except for Local ED 2). ED beds are considered "occupied" only when patients are receiving ED or specialty care, or when they are waiting for specialists. In all three disaster scenarios, more patients are served and discharged at the local facilities in the telemedicine case than in the base case. The reduction in ED bed usage rates from telemedicine in the medium and minor scale scenarios therefore results from the reduction of patient waiting times for local specialty care. As more specialists are reachable locally in the telemedicine case, patients spend less time occupying ED beds waiting for the next available specialist. In other

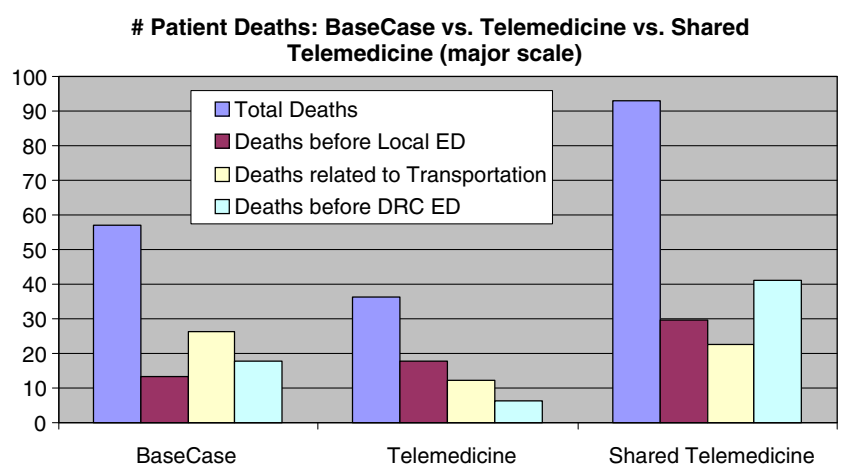

Fig. 6 Patient mortality: base case vs telemedicine vs shared telemedicine (Major Scale) 
Table 2 Number of trauma patients that receive specialty care at the Designated Receiving Center (DRC)

\begin{tabular}{llccccc}
\hline & \# Transferred & \# DOA & $\begin{array}{l}\text { \# Deaths while } \\
\text { Waiting for Specialists }\end{array}$ & $\begin{array}{l}\text { \# Patients that } \\
\text { Receive Specialty Care }\end{array}$ & $\begin{array}{c}\text { \% Patients that Arrive Alive } \\
\text { and Receive Specialty Care }\end{array}$ \\
\hline \multirow{2}{*}{ Major Scale } & Current & 575.17 & 131.51 & 113.83 & 329.83 & $57.34 \%$ \\
& Telemedicine & 543.78 & 120.86 & 92.71 & 330.21 & $60.72 \%$ \\
\hline
\end{tabular}

words, telemedicine helps reduce patients' ED "boarding" times and improve the efficiency of bed usage (that is, more patients served with less total bed usage time).

The local and DRC specialists' "busy rates" are generally higher in the base case than in the TM case, which is expected since in the latter case there are also remote specialists to share their work load. Interestingly, the local specialists are busiest not in the major scale scenario that has the largest incoming demand for specialty care, but in the medium scale scenario. This is likely due to the fact that local EDs are heavily overwhelmed in a major scale event, with ED queue lengths often exceeding the transfer threshold values. As a result succeeding specialty-careseeking patients would "balk" the local ED queues and join the transportation queue instead, even when specialists are locally available and not occupied at the time.

Patient waiting times

The average waiting times for ED beds and specialists at local EDs and at the DRC are both shorter in the TM (telemedicine) setting than in the base cases, as shown in Tables 5 and 6. The TM-enhanced process leads to significant reduction of waiting times for patients' ED bed and specialist encounter. In the major scenario, average waiting times are reduced by hours, and in the medium and minor scaled scenarios, average waiting times are reduced to minimal values.
Table 3 Average usage rates of local resource

\begin{tabular}{|c|c|c|c|c|c|c|c|}
\hline & & \multirow[t]{2}{*}{ Local ED } & \multirow[t]{2}{*}{ ED beds } & \multicolumn{3}{|c|}{ Specialists } & \multirow[t]{2}{*}{ Ambulances } \\
\hline & & & & Trauma & Burn & Other & \\
\hline \multirow[t]{8}{*}{ Major scale } & \multirow[t]{4}{*}{ base case } & 1 & $94.55 \%$ & $50.49 \%$ & - & - & $13.85 \%$ \\
\hline & & 2 & $49.76 \%$ & - & - & - & $28.99 \%$ \\
\hline & & 3 & $97.39 \%$ & $47.18 \%$ & - & $18.66 \%$ & $16.92 \%$ \\
\hline & & 4 & $90.51 \%$ & $46.95 \%$ & - & - & $15.89 \%$ \\
\hline & \multirow[t]{4}{*}{ telemed } & 1 & $90.25 \%$ & $36.04 \%$ & - & - & $11.14 \%$ \\
\hline & & 2 & $96.70 \%$ & - & - & - & $20.26 \%$ \\
\hline & & 3 & $95.16 \%$ & $37.31 \%$ & - & $16.66 \%$ & $12.69 \%$ \\
\hline & & 4 & $90.77 \%$ & $32.72 \%$ & - & - & $13.55 \%$ \\
\hline \multirow[t]{8}{*}{ Medium scale } & \multirow[t]{4}{*}{ base case } & 1 & $91.42 \%$ & $79.78 \%$ & - & - & $6.62 \%$ \\
\hline & & 2 & $34.42 \%$ & - & - & - & $20.70 \%$ \\
\hline & & 3 & $79.31 \%$ & $70.83 \%$ & - & $13.59 \%$ & $5.64 \%$ \\
\hline & & 4 & $74.22 \%$ & $63.80 \%$ & - & - & $6.62 \%$ \\
\hline & \multirow[t]{4}{*}{ telemed } & 1 & $69.36 \%$ & $55.99 \%$ & - & - & $3.54 \%$ \\
\hline & & 2 & $74.32 \%$ & - & - & - & $6.44 \%$ \\
\hline & & 3 & $76.34 \%$ & $59.26 \%$ & - & $17.24 \%$ & $4.28 \%$ \\
\hline & & 4 & $70.92 \%$ & $48.56 \%$ & - & - & $4.39 \%$ \\
\hline \multirow[t]{8}{*}{ Minor scale } & \multirow[t]{4}{*}{ base case } & 1 & $47.13 \%$ & $64.45 \%$ & - & - & $2.43 \%$ \\
\hline & & 2 & $20.10 \%$ & - & - & - & $12.19 \%$ \\
\hline & & 3 & $40.92 \%$ & $50.76 \%$ & - & $5.67 \%$ & $2.39 \%$ \\
\hline & & 4 & $40.74 \%$ & $46.46 \%$ & - & - & $3.03 \%$ \\
\hline & \multirow[t]{4}{*}{ telemed } & 1 & $34.09 \%$ & $40.09 \%$ & - & - & $1.88 \%$ \\
\hline & & 2 & $35.54 \%$ & - & - & - & $3.03 \%$ \\
\hline & & 3 & $38.68 \%$ & $40.77 \%$ & - & $5.90 \%$ & $2.06 \%$ \\
\hline & & 4 & $34.87 \%$ & $31.57 \%$ & - & - & $2.29 \%$ \\
\hline
\end{tabular}


Table 4 Average usage rates of Designated Receiving Center (DRC) and remote resource

\begin{tabular}{|c|c|c|c|c|c|c|c|c|c|}
\hline & & \multirow[t]{2}{*}{ ED Beds } & \multicolumn{3}{|c|}{ DRC specialists } & \multirow[t]{2}{*}{ TeleLink } & \multicolumn{3}{|c|}{ Remote Specialists } \\
\hline & & & Trauma & Burn & Other & & Trauma & Burn & Other \\
\hline \multirow[t]{2}{*}{ Major scale } & basecase & $56.50 \%$ & $52.37 \%$ & $46.82 \%$ & $52.03 \%$ & - & - & - & - \\
\hline & telemed & $29.98 \%$ & $37.20 \%$ & $29.03 \%$ & $36.74 \%$ & $48.73 \%$ & $24.20 \%$ & $19.29 \%$ & $19.53 \%$ \\
\hline \multirow[t]{2}{*}{ Medium scale } & basecase & $8.86 \%$ & $21.63 \%$ & $16.74 \%$ & $16.08 \%$ & - & - & - & - \\
\hline & telemed & $0.69 \%$ & $1.88 \%$ & $1.06 \%$ & $1.15 \%$ & $67.38 \%$ & $40.45 \%$ & $18.41 \%$ & $17.49 \%$ \\
\hline \multirow[t]{2}{*}{ Minor scale } & basecase & $3.37 \%$ & $7.12 \%$ & $12.09 \%$ & $3.58 \%$ & - & - & - & - \\
\hline & telemed & $0.00 \%$ & $0.00 \%$ & $0.00 \%$ & $0.00 \%$ & $29.02 \%$ & $17.24 \%$ & $12.38 \%$ & $3.86 \%$ \\
\hline
\end{tabular}

\section{Discussion and conclusion}

The study presents quantitative results of a simulation study testing the concept of a telemedicine hub (TMH) for patient treatment during the acute phase of emergency response. Our results suggest that a TM-enhanced strategy of preserving local management of disaster victims (i.e., not sending large number to the DRC) may improve the coordination of resources between the receiving center and peripheral treatment facilities, resulting in better health outcomes. With essentially the same resources (i.e. local and DRC ED beds and health care providers), health outcomes are typically better when telemedicine is used in disaster scenarios of various scales. We believe the proposed TM Hub model provides a useful planning and training platform for regional disaster response preparations. Previous work has suggested that the potential roles of telemedicine in disaster situations can be categorized as follows:

(1) Improved situational awareness and communication.

The impact of disasters can be unexpected and extensive, resulting in destruction of ground communications and transportation facilities in addition to casualties. The Mexico City earthquake of September 1985 disrupted all land-based forms of communication in the city, except for a few ham radio operations. Thus the satellite link NASA provided within $24 \mathrm{~h}$ of onset of the disaster was vital for the international rescue and relief efforts [7]. During multiple humanitarian support missions that the SMART
Team, a United States military telemedicine team, carried out in Africa and Pakistan in 2005, they observed that sometimes the most critical tasks were focused on providing communications capabilities, while the amount of actual telemedicine activities may be small due to other limitations [14]. In the response stage of the 2008 Sichuan earthquake, mobile phones and telecommunications equipment were among the first batches of materiel air dropped to the most heavily impacted regions [19].

\section{(2) Assistance in field triage.}

A large natural catastrophe or disaster often results in an overwhelming number of casualties. In such incidents, priority of medical care is often given to initial patient triage, acute care in the field and rapid patient disposition. The ability to obtain accurate information on the victims in a timely manner is critical to the success of continuing medical response [11]. Electronic triage tags, as well as other documental and communicational telemedicine facilities such as hand-held first responder devices (PDA's) and online data centers, have been put to the test in various simulated and actual disaster events $[11,17,21]$. This new telemedical triage system overcomes many limitations of the current paper triage tags; its advantages include real-time data transmission, improved capability for documentation of injuries, improved accuracy for information transfer, weather resistance, etc. As advances in miniaturization of computing and wireless technologies continue, the use of telemedicine for triage in future mass casualty incidents is expected to grow significantly.

Table 5 Average waiting times for ED beds (hours): Base case vs. Telemedicine (TM) Case

\begin{tabular}{|c|c|c|c|c|c|c|c|c|}
\hline \multirow[t]{2}{*}{ Disaster scale } & \multicolumn{4}{|c|}{ Local EDs } & \multicolumn{4}{|l|}{ DRC } \\
\hline & Base & $\mathrm{TM}$ & Reduction & $\%$ Reduction & BaseCase & $\mathrm{TM}$ & Reduction & $\%$ Reduction \\
\hline Major & 34.77 & 32.12 & 2.65 & $7.63 \%$ & 6.22 & 0.39 & 5.83 & $93.69 \%$ \\
\hline Medium & 4.27 & 0.29 & 3.98 & $93.18 \%$ & 0.00 & 0.00 & - & - \\
\hline Minor & 0.14 & 0.01 & 0.12 & $91.09 \%$ & 0.00 & NA & - & - \\
\hline
\end{tabular}


Table 6 Critical patients' average waiting times for ED beds (hours): Base Case vs. Telemedicine (TM) case

\begin{tabular}{|c|c|c|c|c|c|c|c|c|c|}
\hline & \multicolumn{3}{|c|}{ Major scale } & \multicolumn{3}{|c|}{ Medium scale } & \multicolumn{3}{|c|}{ Minor scale } \\
\hline & Base & $\mathrm{TM}$ & $\%$ Reduction & Base & $\mathrm{TM}$ & Reduction & Base & $\mathrm{TM}$ & $\%$ Reduction \\
\hline Local ED 1 & 1.4464 & 0.5212 & $63.97 \%$ & 0.6733 & 0.0629 & $90.66 \%$ & 0.0918 & 0.0052 & $94.34 \%$ \\
\hline Local ED 2 & NA & 1.5386 & NA & NA & 0.2006 & NA & NA & 0.0297 & NA \\
\hline Local ED 3 & 0.5675 & 0.3132 & $44.81 \%$ & 0.1907 & 0.0536 & $71.89 \%$ & 0.0097 & 0.0018 & $81.44 \%$ \\
\hline Local ED 4 & 1.1864 & 0.7033 & $40.72 \%$ & 0.4112 & 0.1097 & $73.32 \%$ & 0.0641 & 0.0123 & $80.81 \%$ \\
\hline DRC & 0.5357 & 0.0742 & $86.15 \%$ & 0 & 0 & $0.00 \%$ & 0 & NA & - \\
\hline
\end{tabular}

(3) Augmentation of local medical surge capacity.

When disasters happen, the capabilities of local medical care facilities can be severely compromised. Local hospitals and clinics may be physically damaged or inaccessible, communication with the outside world may be severed or insufficient, and medical care givers may be unable to reach the scene due to the effects of the disaster. Even with infrastructure intact, various types of injuries resulting from the disaster may require medical specialists that are not available in the affected region; in addition, the sudden surge casualties and overwhelming demands for care present a serious challenge to the effectiveness of medical response. Telemedicine can be used to provide external help in a relatively short time frame. In addition to assisting with pre-hospital management such as initial triage, previous reports document other ways to rapidly deploy telemedical capabilities, such as in mobile field hospitals [14] and via email and video consultations [10, 20]. In one case, remote physicians were virtually brought to scene via telemedicine to boost the medical capacity of doctors and first responders in the field for providing a range of care including diagnosis, treatment, monitoring, etc. [3].

(4) Enhanced planning and preparedness.

The community involved in disaster response activities have come to realize that the effectiveness of disaster relief efforts, including those of a telemedical nature, depends heavily on the availability, accessibility and deployability of various resources including people (such as responders and physicians), infrastructure (such as equipments for communications), information technology (mobile communications devices, base stations, GIS, online databases, expert systems), and reserves of resupply materiel (e.g., batteries). The resources need to be prepositioned or rapidly deployable in order to be utilized at the time of crisis. Numerous planning groups have been formed, and drills exercised, often via advanced virtual reality technologies, to establish and evaluate the resource requirements, data gaps, and the efficacy of process management structures to enable effective telemedicine capability [7, 20, 29].
(5) Delivery of post-response support, rehabilitation and education

In the aftermath of a disaster, telemedicine can continually provide post-traumatic patient management and support such as telemonitoring, telepsychiatric care and telerehabilitation education for the affected population. Surveys indicate that applications in this area are most encouraging; they can be more cost-effective for both care providers and patients [26], and the quality can be comparable to that of the traditional means [28]. In some reported cases, patient care was considered improved due to customizable telerehabilitation programs [4]. Existing telemedicine networks and facilities also provide a platform for disaster-related research, such as information sharing, tele-education and e-training for injury types, disaster epidemiology, and medical management strategies.

In all the scenarios we considered, we observe that application of telemedicine helps local EDs serve more patients locally while maintaining lower ED bed occupation rates by reducing patients' waiting (boarding) times, hence utilizing resources more efficiently. On the other hand, properly functioning TMHs will require rapid access to external specialists for optimal performance. That is, the benefits of telemedicine in heavy demand disaster scenarios require the rapid availability of external specialists, stressing the need to establish and maintain such resources for emergent uses.

For these external remote specialists to contribute to the emergency response operation, they need to be part of a regional telemedicine network that can be quickly accessed and put to use during crises. If such a network has not been established or properly maintained, emergency telemedicine support to local disaster areas may have to be sought manually via coordinators from the center. This working process, however feasible in regular times, may result in aggravated demand on the coordinators and processes at the center, as well as deteriorating system performance, especially for scenarios of major scale disasters with heavy demand at the center already.. We cannot assume that telemedicine without external specialists would still result in improvements in the demand-intensive scenario. 
We have developed a model that is generally applicable to the design and functioning of telemedicine systems for disaster response. However, it has several important limitations. First, we did not model the incorporation of, and impact of telemedicine activities on, command-andcontrol systems such as ICS as well as other disaster response systems such as EMS. Second, the model requires a number of assumptions about patients' arrival patterns at the local facilities, injury types that may present, scales of patient's injury severity, as well as representation of various treatment processes and time-dependent mortality. Although the simulation is able to describe the overall medical response process (sequence of events and resource requirements), more complex models will need to be constructed and to represent more complicated issues that arise in reality. Last, the simulation presented here is an analytical tool, but not an optimization tool. It may be used to evaluate the performance of alternatives (such as different queue length threshold values for transfer rules) in various scenarios. However, it is not able to directly provide a solution, or suggest a policy, that can be used to guide the operations and routing directions within the process. This model shows that existing telemedicine technology can be applied to current disaster response activities to enhance surge capacity of the healthcare system and the speed and effectiveness of medical response, to facilitate communications and improve resource and operations planning, and to increase situational awareness within the command and control system and overall community.

Funding Grant 1 HSPEP070013-01-00 (Surge Capacity Through Technology Innovation), Principal Investigator: Christian Sandrock, UC Davis.

Open Access This article is distributed under the terms of the Creative Commons Attribution Noncommercial License which permits any noncommercial use, distribution, and reproduction in any medium, provided the original author(s) and source are credited.

\section{References}

1. ABCRadioAustralia (2005) Telemedicine Aids Tsunami Victims. from http://www.abc.net.au/ra/innovations/stories/s1343400.htm

2. Doarn, C. R., Lavrentyev, V. A., et al., Evolution of telemedicine in Russia: the influence of the space program on modern telemedicine programs. Telemed J E Health 9(1):103-9, 2003.

3. Felland, L. E., Katz, A., et al., Developing health system surge capacity: community efforts in jeopardy. Res Briefs 5:1-8, 2008.

4. Galea, M., Tumminia, J., et al., Telerehabilitation in spinal cord injury persons: a novel approach. Telemed J E Health 12(2):160-2, 2006.

5. Ganjouei, et al., The pattern of injuries among the victims of the Bam earthquake. Iran J Publ Health 37(3):70-76, 2008.

6. Garner, A., Lee, A., et al., Comparative analysis of multiplecasualty incident triage algorithms. Ann Emerg Med 38(5):541-8, 2001.
7. Garshnek, V., and Burkle, F. M., Jr., Applications of telemedicine and telecommunications to disaster medicine: historical and future perspectives. J Am Med Inform Assoc 6(1):26-37, 1999.

8. Garshnek, V., Logan, J. S., et al., The telemedicine frontier: going the extra mile. Space Pol 13(1):37-46, 1997.

9. Hupert, N., Hollingsworth, E., et al., Is overtriage associated with increased mortality? Insights from a simulation model of mass casualty trauma care. Disaster Med Public Health Prep 1(1 Suppl):S14-24, 2007.

10. Jamal, A., Gilani, S., et al., Role of telemedicine during disaster: a case study. J Rawalpindi Med Coll 10(2):94-96, 2005.

11. Killeen JP, TC Chan, et al. (2006) A wireless first responder handheld device for rapid triage, patient assessment and documentation during mass casualty incidents. AMIA Annu Symp Proc, pp 429-33.

12. Lach JM, Vazquez RM (2005) Simulation model of the telemedicine program. Proceedings of the 2004 Winter Simulation Conference

13. Mack D, Brantley, KM, et al. (2007) Mitigating the health effects of disasters for medically underserved populations: electronic health records, telemedicine, research, screening, and surveillance. J Health Care Poor Underserved 18(2)

14. Meade, K., and Lam, D. M., A deployable telemedicine capability in support of humanitarian operations. Telemed $J E$ Health 13 (3):331-40, 2007.

15. Melnick GA, Nawathe, AC, et al. (2004) Emergency department capacity and access in California, 1990-2001: an economic analysis. Health Aff (Millwood) Suppl Web Exclusives: W4-136-42

16. Memarzadeh, M., Loghmani, A., et al., The field hospital setting in earthquake. J Res Med Sci 9(5):199-204, 2004.

17. Paunksnis, A., Barzdziukas, V., et al., An assessment of telemedicine possibilities in massive casualties situations. Rocz Akad Med Bialymst 50:201-3, 2005.

18. Peek-Asa, C., Kraus, J. F., et al., Fatal and hospitalized injuries resulting from the 1994 Northridge earthquake. Int J Epidemiol 27 (3):459-65, 1998.

19. People's Daily (2008) Army airdrops disaster relief goods to Sichuan. People's Daily Online, from http://english.peopledaily. com.cn/90001/90776/90882/6410865.html.

20. Pieper, B., and Meineke, V., Nuclear medical expertise delivered by telemedicine in a 'dirty bomb' exercise. J Telemed Telecare 13 (3):154-6, 2007.

21. Plischke, M., Wolf, K. H., et al., Telemedical support of prehospital emergency care in mass casualty incidents. Eur $J$ Med Res 4(9):394-8, 1999.

22. Sacco, W. J., Navin, D. M., et al., Precise formulation and evidence-based application of resource-constrained triage. Acad Emerg Med 12(8):759-70, 2005.

23. Sampalis, J. S., Denis, R., et al., Trauma care regionalization: a processoutcome evaluation. J Trauma 46(4):565-79, 1999. discussion 579-81.

24. Sastrokusumo U, Suksmono AB, et al. (2005) Contribution for post-tsunami syndrome: multimedia application in medical teleeducation and telemedicine. 7th International Workshop on Enterprise networking and Computing in Healthcare Industry

25. Schultz CH, Koenig KL, Noji EK. A medical disaster response to reduce immediate mortality after an earthquake. NEJM 1996; 334:438-444.

26. Shore, J. H., Brooks, E., et al., An economic evaluation of telehealth data collection with rural populations. Psychiatr Serv 58 (6):830-5, 2007.

27. Simmons SC, Hamilton DR, et al. (2008) telemedicine. Principles of Clinical Medicine for Space Flight. M. Barratt and S. Pool, Springer

28. Whitten, P., and Love, B., Patient and provider satisfaction with the use of telemedicine: overview and rationale for cautious enthusiasm. J Postgrad Med 51(4):294-300, 2005.

29. Yellowlees, P., Cook, J. N., et al., Can virtual reality be used to conduct mass prophylaxis clinic training? A pilot program. Biosecur Bioterror 6(1):36-44, 2008. 